\title{
Dependent Insurance Risk Model: Deterministic Threshold
}

\author{
Isaac K. M. Kwan and Hailiang Yang*
}

\begin{abstract}
This paper considers a dependent insurance risk model. We assume that the inter-arrival time depends on the previous claim size through a deterministic threshold structure. Adjustment coefficient and Lundberg type upper bound for the ruin probability are obtained. In case of exponential claim size, an explicit solution for the ruin probability is obtained by solving a system of ordinary delay differential equations. Some numerical results are included for illustration purposes.
\end{abstract}

Key Words: Ruin probability, Lundberg inequality, exponential claim distribution, delay differential equation.

*Department of Statistics and Actuarial Science, The University of Hong Kong, Pokfulam Road, Hong Kong, e-mails: kwokman ${ }_{k} w a n @ y a h o o . c o m . h k(K w a n), h l y a n g @ h k u s u a . h k u . h k(Y a n g)$ 


\section{Introduction}

Insurance risk models with dependent structure have become an important research area in actuarial science, and more and more researchers are interested in this topic nowadays. In the classical insurance risk model, the assumptions that the successive claims are independent and identically distributed (i.i.d.), and that the number of claims follows a Poisson or renewal process seem too restrictive. The claim frequencies and severities for automobile insurance and life insurance are not altogether independent. Different models have been proposed to relax such restrictions. The simplest dependence model is the discrete time autoregressive model in the standard text book Bowers et al. (1997). For a detailed discussion on the dependent insurance risk models, we refer the readers to the papers by Dhaene and Denuit (1999), Dhaene and Goovaverts (1996) and Dhaene et al. (2002a, 2002b) and the references therein. Albrecher and Boxma (2004) consider a ruin model with a dependent setting, where an inter-arrival time depends on the previous claim size with a random threshold. In their paper, they derive the Laplace transform of the ultimate ruin probability. They use a system of simultaneous equations to find the value of the ultimate ruin probability when the initial surplus is zero. By inverting the Laplace transform, theoretically it is possible to obtain the ultimate ruin probability. However, in general, it is not possible to obtain an explicit solution of the inversion of the Laplace transform.

In this paper, we consider a similar model to that in Albrecher and Boxma (2004), but a fixed threshold. We define the adjustment coefficient for this model and obtain the Lundberg type upper bound for the ruin probability. When the claim size follows an exponential distribution, by solving a system of delay differential equations, an 
explicit solution for the ruin probability is obtained. Some numerical examples are presented in the last section.

\section{The Insurance Risk Model}

In the model discussed in Albrecher and Boxma (2004), the distribution of interarrival time depends on the previous claim size. Let $X_{k}$ be the size of the $\mathrm{k}$-th claim, $T_{k}$ be the inter-arrival time between the (k-1)-th claim and the $\mathrm{k}$-th claim. If $X_{k-1}$ is less than a threshold level $a$, then $T_{k}$ follows an exponential distribution with parameter $\beta_{1}$. Otherwise, it follows an exponential distribution with different parameter $\beta_{2}$. Albrecher and Boxma (2004) assume that the threshold level $a$ is an exponential distributed random variable. In this paper, we assume that $a$ is a constant.

We assume that claim sizes are i.i.d. with common mean $\mu$. Let $U_{t}(u)$ be the surplus process at time $t$ given the initial surplus $u$, then we have

$$
U_{t}(u)=u+c t-S_{t},
$$

where $S_{t}=\sum_{k=1}^{N(t)} X_{k}$ and $c$ is a constant premium rate. In our model, if $X_{k-1}<a$, then $T_{k} \sim \exp \left(\beta_{1}\right)$; and if $X_{k-1} \geq a$, then $T_{k} \sim \exp \left(\beta_{2}\right)$.

Let us assume the following net profit condition is satisfied:

$$
\mu<c\left[\frac{P(X<a)}{\beta_{1}}+\frac{P(X \geq a)}{\beta_{2}}\right] .
$$


Let $T=i n f_{t \geq 0}\left\{t: U_{t}(u)<0\right\}$ be the time of ruin, then $\phi_{i}(u)=P\{T=\infty \mid U(0)=$ $\left.u, T_{1} \sim \exp \left(\beta_{i}\right)\right\}$ and $\psi_{i}(u)=1-\phi_{i}(u)$ are the ultimate survival probability and the ultimate ruin probability given the first claim occurs according to the exponential distribution with parameters $\beta_{i},(i=1,2)$, respectively.

The following results are the same as in Albrecher and Boxma (2004), which provide the integro-differential equation and the Laplace transform of the ultimate ruin probability.

Lemma 2.1 Let $f(x)$ be the density function of the claim size random variable. The ultimate survival probability $\phi_{i}(u)(i=1,2)$ satisfies the following integro-differential equation:

$$
\begin{aligned}
c \frac{d \phi_{i}(u)}{d u}= & \beta_{i} \phi_{i}(u)-\beta_{i} \int_{0}^{u} I(a>y) \phi_{1}(u-y) f(y) d y \\
& -\beta_{i} \int_{0}^{u} I(a \leq y) \phi_{2}(u-y) f(y) d y .
\end{aligned}
$$

Lemma 2.2 Let $\widetilde{\phi}_{i}(s)$ be the Laplace transform of $\phi_{i}(u)(i=1,2)$. Define:

$$
\begin{gathered}
\chi_{1}(s)=\int_{0}^{\infty} e^{-s x} I(a>x) f(x) d x=\int_{0}^{a} e^{-s x} f(x) d x \\
\chi_{2}(s)=\int_{0}^{\infty} e^{-s x} I(a \leq x) f(x) d x=\int_{a}^{\infty} e^{-s x} f(x) d x .
\end{gathered}
$$

Then,

$$
\widetilde{\phi}_{1}(s)=\frac{c \phi_{1}(0)\left[c s-\beta_{2}+\beta_{2} \chi_{2}(s)\right]-c \beta_{1} \chi_{2}(s) \phi_{2}(0)}{\left[c s-\beta_{2}+\beta_{2} \chi_{2}(s)\right]\left[c s-\beta_{1}+\beta_{1} \chi_{1}(s)\right]-\beta_{1} \beta_{2} \chi_{1}(s) \chi_{2}(s)}
$$


and,

$$
\widetilde{\phi}_{2}(s)=\frac{c \phi_{2}(0)\left[c s-\beta_{1}+\beta_{1} \chi_{1}(s)\right]-c \beta_{2} \chi_{1}(s) \phi_{1}(0)}{\left[c s-\beta_{2}+\beta_{2} \chi_{2}(s)\right]\left[c s-\beta_{1}+\beta_{1} \chi_{1}(s)\right]-\beta_{1} \beta_{2} \chi_{1}(s) \chi_{2}(s)} .
$$

The initial values $\phi_{1}(0)$ and $\phi_{2}(0)$ can be obtained by solving the following system of simultaneous equations:

$$
\left\{\begin{array}{l}
\left(1-\phi_{1}(0)\right) \frac{F(a)}{\beta_{1}}+\left(1-\phi_{2}(0)\right) \frac{1-F(a)}{\beta_{2}}=\frac{\mu}{c} \\
\phi_{1}(0)=\frac{c \sigma-\beta_{1}+\beta_{1} \chi_{1}(\sigma)}{\beta_{2} \chi_{1}(\sigma)} \phi_{2}(0)
\end{array}\right.
$$

where $\sigma$ is a unique root with positive real part satisfying $\left[c s-\beta_{2}+\beta_{2} \chi_{2}(s)\right][c s-$ $\left.\beta_{1}+\beta_{1} \chi_{1}(s)\right]-\beta_{1} \beta_{2} \chi_{1}(s) \chi_{2}(s)=0$.

Using $\phi_{1}(0)$ and $\phi_{2}(0)$ together with $(2)$ and $(3), \widetilde{\phi}_{1}(s)$ and $\widetilde{\phi}_{2}(s)$ can be completely determined. Theoretically $\phi_{1}(u)$ and $\phi_{2}(u)$ can be obtained by inverting the Laplace transforms.

\section{Adjustment Coefficient and Lundberg Inequal- ity}

In this section, we define the adjustment coefficient and derive the Lundberg type upper bound for ruin probability in our model.

Theorem 3.1 Assume that $\hat{m}_{X}(r)$, the moment generating function of the claim size $X$, exists and that there exists $r_{\infty} \in \mathbb{R} \bigcup\{\infty\}$ such that $\hat{m}_{X}(r)<\infty$ if $r<r_{\infty}$ and 
$\lim _{r \rightarrow r_{\infty}} \hat{m}_{X}(r)=\infty$, then the equation $E\left[e^{r(X-c T)}\right]=1$ has a unique positive solution $R$. $R$ is called adjustment coefficient.

\section{Proof}

$$
\begin{aligned}
E\left[e^{r(X-c T)}\right] & =E\left[e^{r(X-c T)} ; X<a\right]+E\left[e^{r(X-c T)} ; X \geq a\right] \\
& =\frac{\beta_{1}}{\beta_{1}+r c} E\left[e^{r X} ; X<a\right]+\frac{\beta_{2}}{\beta_{2}+r c} E\left[e^{r X} ; X \geq a\right] \\
& =\frac{\beta_{1}}{\beta_{1}+r c} \int_{0}^{a} e^{r x} f(x) d x+\frac{\beta_{2}}{\beta_{2}+r c} \int_{a}^{\infty} e^{r x} f(x) d x .
\end{aligned}
$$

Let

$$
g(r)=\frac{\beta_{1}}{\beta_{1}+r c} \chi_{1}(-r)+\frac{\beta_{2}}{\beta_{2}+r c} \chi_{2}(-r) .
$$

It is obvious that $g(0)=1$.

$$
\begin{aligned}
g^{\prime}(r)= & -\frac{\beta_{1} c}{\left(\beta_{1}+r c\right)^{2}} \int_{0}^{a} e^{r x} f(x) d x+\frac{\beta_{1}}{\beta_{1}+r c} \int_{0}^{a} x e^{r x} f(x) d x \\
& -\frac{\beta_{2} c}{\left(\beta_{2}+r c\right)^{2}} \int_{a}^{\infty} e^{r x} f(x) d x+\frac{\beta_{2}}{\beta_{2}+r c} \int_{a}^{\infty} x e^{r x} f(x) d x
\end{aligned}
$$

The first derivative of $g(r)$ at 0 is

$$
\begin{aligned}
& g^{\prime}(0)=-\frac{c}{\beta_{1}} \int_{0}^{a} f(x) d x+\int_{0}^{a} x f(x) d x-\frac{c}{\beta_{2}} \int_{a}^{\infty} f(x) d x+\int_{a}^{\infty} x f(x) d x \\
= & \mu-\frac{c}{\beta_{1}} \int_{0}^{a} f(x) d x-\frac{c}{\beta_{2}} \int_{a}^{\infty} f(x) d x=\mu-c\left[\frac{P(X<a)}{\beta_{1}}+\frac{P(X \geq a)}{\beta_{2}}\right]<0,
\end{aligned}
$$

where the last inequality is due to the net profit condition. Moreover, $g(r) \rightarrow \infty$ as $r \rightarrow r_{\infty}$ and $g^{\prime \prime}(r)=E\left[(X-c T)^{2} e^{r(X-c T)}\right]>0, \forall r>0$. So, there exists a unique positive number $R$ such that $g(R)=1$.

With the adjustment coefficient defined, Lundberg's Inequality can be obtained as follows: 
Theorem 3.2 (Lundberg's Inequality)

$$
\forall u \geq 0, \quad \psi_{i}(u) \leq \frac{\beta_{i}}{\beta_{i}+R c} e^{-R u}, \quad i=1,2,
$$

where $R$ is the adjustment coefficient.

Proof Let $S_{n}$ be a random walk with identical and independent increments $Y_{k}=$ $X_{k}-c T_{k+1}$. Then the classical technique of changing measure can be applied to our model too. By a similar argument to that in Asmussen (2000), and defining a new probability measure by $P_{L}(A)=E\left[e^{R S_{n}} ; A\right]$, we have

$$
\begin{aligned}
\psi_{i}(u) & =E\left[E_{L}\left[e^{-R S_{\tau\left(u+c T_{1}\right)}} \mid T_{1}\right] \mid T_{1} \sim \exp \left(\beta_{i}\right)\right] \\
& =E\left[e^{-R\left(u+c T_{1}\right)} E_{L}\left[e^{-R \xi\left(u+c T_{1}\right)} \mid T_{1}\right] \mid T_{1} \sim \exp \left(\beta_{i}\right)\right] \\
& \leq E\left[e^{-R\left(u+c T_{1}\right)} \mid T_{1} \sim \exp \left(\beta_{i}\right)\right] \\
& =\frac{\beta_{i}}{\beta_{i}+R c} e^{-R u}
\end{aligned}
$$

where $\xi(u)$ is the first overshoot amount.

\section{Exponential Claim Size Distribution}

Suppose the distribution of claim size follows an exponential distribution, i.e. $F(x)=$ $1-e^{-\lambda x}$. It can be seen that to obtain the explicit solution for the ultimate ruin probability is difficult from inverting the Laplace transform in this model. In order to have some insight into the dynamic behaviour of the ultimate ruin probability, we derive the differential equation satisfied by the ruin probability. 


\subsection{Coupled System of Second Order Linear Delay Differen- tial Equations}

This section obtains the following system of delay ODEs for the ruin probability.

Theorem $4.1 \psi_{1}(u)$ and $\psi_{2}(u)$ satisfy the following coupled systems of second order linear delay differential equations:

$$
\left\{\begin{array}{l}
c \frac{d^{2} \psi_{1}(u)}{d u^{2}}+\left(\lambda c-\beta_{1}\right) \frac{d \psi_{1}(u)}{d u}+\beta_{1} \lambda e^{-\lambda a}\left[\psi_{2}(u-a)-\psi_{1}(u-a)\right]=0, \quad u>a \\
c \frac{d^{2} \psi_{2}(u)}{d u^{2}}+\left(\lambda c-\beta_{2}\right) \frac{d \psi_{2}(u)}{d u}+\beta_{2} \lambda\left[\psi_{1}(u)-\psi_{2}(u)\right] \\
+\beta_{2} \lambda e^{-\lambda a}\left[\psi_{2}(u-a)-\psi_{1}(u-a)\right]=0, \quad u>a
\end{array}\right.
$$

and

$$
\left\{\begin{array}{lll}
c \frac{d^{2} \psi_{1}(u)}{d u^{2}}+\left(\lambda c-\beta_{1}\right) \frac{d \psi_{1}(u)}{d u} & =0, & 0 \leq u \leq a \\
c \frac{d^{2} \psi_{2}(u)}{d u^{2}}+\left(\lambda c-\beta_{2}\right) \frac{d \psi_{2}(u)}{d u}+\lambda \beta_{2}\left(\psi_{1}(u)-\psi_{2}(u)\right) & =0, & 0 \leq u \leq a
\end{array}\right.
$$

Proof From (1), since the equation depends on whether $u$ is less or greater than the threshold $a$, we break down the proof into 2 cases:

Case 1: $u \leq a$ : In this case (1) becomes

$$
c \frac{d \phi_{1}(u)}{d u}=\beta_{1} \phi_{1}(u)-\beta_{1} \int_{0}^{u} \phi_{1}(u-y) \lambda e^{-\lambda y} d y .
$$

Changing the variable by $x=u-y$, we have

$$
c \frac{d \phi_{1}(u)}{d u}=\beta_{1} \phi_{1}(u)-\beta_{1} \lambda e^{-\lambda u} \int_{0}^{u} \phi_{1}(x) e^{\lambda x} d x
$$


Differentiating both sides with respect to $u$, we have

$$
c \frac{d^{2} \phi_{1}(u)}{d u^{2}}=\beta_{1} \frac{d \phi_{1}(u)}{d u}-\beta_{1} \lambda \phi_{1}(u)+\beta_{1} \lambda^{2} e^{-\lambda u} \int_{0}^{u} \phi_{1}(x) e^{\lambda x} d x
$$

$\lambda \times(8)+(9)$, we obtain

$$
c \frac{d^{2} \phi_{1}(u)}{d u^{2}}+\lambda c \frac{d \phi_{1}(u)}{d u}=\beta_{1} \frac{d \phi_{1}(u)}{d u}-\beta_{1} \lambda \phi_{1}(u)+\lambda \beta_{1} \phi_{1}(u)
$$

so

$$
c \frac{d^{2} \phi_{1}(u)}{d u^{2}}+\left(\lambda c-\beta_{1}\right) \frac{d \phi_{1}(u)}{d u}=0,
$$

therefore, the first equation in (7) is proved.

Using similar arguments, we can show that, for $u \leq a, \psi_{2}(u)$ satisfies the second equation in (7).

Case 2: $u>a$ : In this case, (1) becomes

$$
c \frac{d \phi_{1}(u)}{d u}=\beta_{1} \phi_{1}(u)-\beta_{1} \int_{0}^{a} \phi_{1}(u-y) \lambda e^{-\lambda y} d y-\beta_{1} \int_{a}^{u} \phi_{2}(u-y) \lambda e^{-\lambda y} d y .
$$

Changing the variable by $x=u-y$, we have

$$
c \frac{d \phi_{1}(u)}{d u}=\beta_{1} \phi_{1}(u)-\beta_{1} \lambda e^{-\lambda u} \int_{u-a}^{u} \phi_{1}(x) e^{\lambda x} d x-\beta_{1} \lambda e^{-\lambda u} \int_{0}^{u-a} \phi_{2}(x) e^{\lambda x} d x .
$$

Differentiating both sides with respect to $u$, we obtain

$$
\begin{aligned}
c \frac{d^{2} \phi_{1}(u)}{d u^{2}}= & \beta_{1} \frac{d \phi_{1}(u)}{d u}-\beta_{1} \lambda e^{-\lambda u}\left[\phi_{1}(u) e^{\lambda u}-\phi_{1}(u-a) e^{\lambda(u-a)}\right] \\
& +\beta_{1} \lambda^{2} e^{-\lambda u} \int_{u-a}^{u} \phi_{1}(x) e^{\lambda x} d x-\beta_{1} \lambda e^{-\lambda u}\left[\phi_{2}(u-a) e^{\lambda(u-a)}\right] \\
& +\beta_{1} \lambda^{2} e^{-\lambda u} \int_{0}^{u-a} \phi_{2}(x) e^{\lambda x} d x
\end{aligned}
$$


$\lambda \times(10)+(11)$, we have

$$
\begin{aligned}
c \frac{d^{2} \phi_{1}(u)}{d u^{2}}+\lambda c \frac{d \phi_{1}(u)}{d u}= & \beta_{1} \frac{d \phi_{1}(u)}{d u}-\beta_{1} \lambda \phi_{1}(u)+\beta_{1} \lambda e^{-\lambda a} \phi_{1}(u-a) \\
& -\beta_{1} \lambda e^{-\lambda a} \phi_{2}(u-a)+\lambda \beta_{1} \phi_{1}(u),
\end{aligned}
$$

therefore,

$$
c \frac{d^{2} \phi_{1}(u)}{d u^{2}}+\left(\lambda c-\beta_{1}\right) \frac{d \phi_{1}(u)}{d u}+\beta_{1} \lambda e^{-\lambda a}\left[\phi_{2}(u-a)-\phi_{1}(u-a)\right]=0 .
$$

From this the first equation in (6) is proved.

Similarly, we can show that, for $u>a, \psi_{2}(u)$ satisfies the second equation in (6). This completes the proof of Theorem 4.1.

\subsection{Explicit Solution}

In this subsection, we derive the explicit expression for the ruin probability by solving (6) and (7) in Section 4.1. In order to do so, the coupled second order linear delay differential equations (6) and (7) should be transformed to systems of first order linear delay differential equation by proper substitutions as follows:

$$
x_{1}(u)=\frac{d \psi_{1}(u)}{d u}, \quad x_{2}(u)=\psi_{1}(u), \quad x_{3}(u)=\frac{d \psi_{2}(u)}{d u}, \quad x_{4}(u)=\psi_{2}(u) .
$$


Define:

$$
\begin{aligned}
& \mathbf{A}=\left(\begin{array}{cccc}
\left(\frac{\beta_{1}}{c}-\lambda\right) & 0 & 0 & 0 \\
1 & 0 & 0 & 0 \\
0 & -\frac{\beta_{2}}{c} \lambda & \frac{\beta_{2}}{c}-\lambda & \frac{\beta_{2}}{c} \lambda \\
0 & 0 & 1 & 0
\end{array}\right) \\
& \mathbf{B}=\left(\begin{array}{cccc}
0 & \frac{\beta_{1}}{c} \lambda e^{-\lambda a} & 0 & -\frac{\beta_{1}}{c} \lambda e^{-\lambda a} \\
0 & 0 & 0 & 0 \\
0 & \frac{\beta_{2}}{c} \lambda e^{-\lambda a} & 0 & -\frac{\beta_{2}}{c} \lambda e^{-\lambda a} \\
0 & 0 & 0 & 0
\end{array}\right), \\
& \mathbf{x}(\mathrm{u})=\left(\begin{array}{c}
x_{1}(u) \\
x_{2}(u) \\
x_{3}(u) \\
x_{4}(u)
\end{array}\right), \quad \mathbf{x}_{\mathbf{0}}=\left(\begin{array}{c}
\psi_{1}^{\prime}(0) \\
\psi_{1}(0) \\
\psi_{2}^{\prime}(0) \\
\psi_{2}(0)
\end{array}\right) .
\end{aligned}
$$

Then (6) and (7) is equivalent to the following first order linear delay differential equations:

$$
\left\{\begin{array}{l}
\mathbf{x}^{\prime}(u)=\mathbf{A} \mathbf{x}(u)+\mathbf{B} \mathbf{x}(u-a) \quad, u>a \\
\mathbf{x}^{\prime}(u)=\mathbf{A} \mathbf{x}(u), \quad u \leq a \\
\mathbf{x}(0)=\mathbf{x}_{\mathbf{0}}
\end{array}\right.
$$

For $u \leq a$, the second equation in (12) is a system of ordinary differential equations. The solution is:

$$
\begin{aligned}
& \psi_{1}(u)=k_{1}+k_{2} e^{\left(\frac{\beta_{1}}{c}-\lambda\right) u} \\
& \psi_{2}(u)=k_{3} e^{-\lambda u}+k_{4} e^{\frac{\beta_{2}}{c} u}+k_{1}+b k_{2} e^{\left(\frac{\beta_{1}}{c}-\lambda\right) u}
\end{aligned}
$$


where,

$$
\begin{aligned}
k_{1} & =\frac{\lambda c \psi_{1}(0)-\beta_{1}}{\lambda c-\beta_{1}}, \quad k_{2}=\frac{\beta_{1}\left(1-\psi_{1}(0)\right)}{\lambda c-\beta_{1}}, \\
k_{3} & =\frac{\beta_{2}-\beta_{2} k_{1}+\left(\beta_{1}-\lambda c-\beta_{2}\right) b k_{2}}{\lambda c+\beta_{2}}, \quad k_{4}=\psi_{2}(0)-k_{3}-k_{1}-b k_{2}, \\
b & =\frac{\lambda \beta_{2}}{\lambda \beta_{2}+c\left(\frac{\beta_{2}}{c}-\lambda\right)\left(\frac{\beta_{1}}{c}-\lambda\right)-c\left(\frac{\beta_{1}}{c}-\lambda\right)^{2}},
\end{aligned}
$$

with $\psi_{1}(0)$ and $\psi_{2}(0)$ found by the method described in Section 2.

Define:

$$
\varphi(u)=\left(\begin{array}{c}
k_{2}\left(\frac{\beta_{1}}{c}-\lambda\right) e^{\left(\frac{\beta_{1}}{c}-\lambda\right) u} \\
k_{1}+k_{2} e^{\left(\frac{\beta_{1}}{c}-\lambda\right) u} \\
-k_{3} \lambda e^{-\lambda u}+k_{4} \frac{\beta_{2}}{c} e^{\frac{\beta_{2}}{c} u}+b k_{2}\left(\frac{\beta_{1}}{c}-\lambda\right) e^{\left(\frac{\beta_{1}}{c}-\lambda\right) u} \\
k_{3} e^{-\lambda u}+k_{4} e^{\frac{\beta_{2}}{c} u}+k_{1}+b k_{2} e^{\left(\frac{\beta_{1}}{c}-\lambda\right) u}
\end{array}\right), \quad 0 \leq u \leq a .
$$

So the system (12) can be reformulated as:

$$
\left\{\begin{array}{l}
\mathbf{x}^{\prime}(u)=\mathbf{A} \mathbf{x}(u)+\mathbf{B} \mathbf{x}(u-a), \quad u>a \\
\mathbf{x}(u)=\varphi(u), \quad 0 \leq u \leq a
\end{array}\right.
$$

It is well-known that the solution of the above system exists and is unique (see Driver, 1977). In general, the explicit form of the solution of a delay differential equation is not easy to obtain. Numerical methods such as Runge-Kutta methods for delay differential equations can be applied to obtain approximated values of the solution (see Bellen and Zennaro, 2003). Thanks to Tsoi (1975), for equations like (13), the explicit solution can be found.

Substituting $\mathbf{y}\left(\frac{u}{a}-1\right)=\mathbf{x}(u)$ and $t=\frac{u}{a}-1$, the system (13) can be converted 
into the following form:

$$
\left\{\begin{array}{l}
\mathbf{y}^{\prime}(t)=a \mathbf{A y}(t)+a \mathbf{B y}(t-1), \quad t>0 \\
\mathbf{y}(t)=\varphi(a(t+1)), \quad-1 \leq t \leq 0
\end{array}\right.
$$

From Tsoi (1975), the solution of the system (14) is given by:

$$
\mathbf{y}(t)=\mathbf{Y}(t) \mathbf{y}(0)+\int_{-1}^{0} \mathbf{Y}(t-\tau-1) a \mathbf{B} \varphi(a(\tau+1)) \mathrm{d} \tau, \quad \mathrm{t}>0
$$

where $\mathbf{Y}(t)$ is a fundamental matrix expressed in the form:

$$
\mathbf{Y}(t)=\sum_{j=0}^{\infty} \sum_{k=j}^{\infty} \frac{\mathbf{1}}{k !} \mathbf{P}_{j+1}^{k+1}(t-j)^{k}
$$

where $\mathbf{P}_{j}^{k}$ satisfies a recursive equation of the form:

$$
\mathbf{P}_{j+1}^{k+1}=a \mathbf{A} \mathbf{P}_{j+1}^{k}+a \mathbf{B} \mathbf{P}_{j}^{k}, \quad j=0,1,2,3, \ldots, \quad k=0,1,2,3, \ldots,
$$

with

$$
\mathbf{P}_{1}^{1}=\mathbf{I}
$$

and

$$
\mathbf{P}_{j}^{k}=0, \quad \text { if } j=0 \text { or } k=0 \text { or } j>k .
$$

Finally, $\mathbf{x}(u)$ can be calculated from $\mathbf{x}(u)=\mathbf{y}\left(\frac{u}{a}-1\right)$.

\section{Numerical Illustration}

In this section we provide a numerical illustration. The following values are used: $\lambda=1, \beta_{1}=1, \beta_{2}=0.1, a=1.0,2.0,5.0, c=\frac{10}{\left\{\lambda\left[\frac{\left(1-e^{-\lambda a}\right)}{\beta_{1}}+\frac{e^{-\lambda a}}{\beta_{2}}\right]\right\}^{2}}$. 
For comparison purpose, the inversion of Laplace Transform suggested by Albrecher and Boxma (2004) as in Section 2, the explicit formula (15) obtained by using the method in Tsoi (1975), and the 1-stage Runge-Kutta numerical methods of order 2 are applied to find the solution of (13), respectively.

We use the FORTRAN programming language and the IMSL package. When we invert the Laplace transform, the INLAP procedure with relative error $=0.01$ is used. The summation in formula (16) was modified to

$$
\sum_{j=0}^{20} \sum_{k=j}^{150} \frac{1}{k !} \mathbf{P}_{j+1}^{k+1}(t-j)^{k}
$$

in our computer programme. Moreover, the DQDAG procedure with IRULE $=2$ is used to perform the integration in (15). The recursive equation of 1-stage RungeKutta methods of order 2 is applied in the following way:

$$
\mathbf{x}_{\mathbf{n}+\mathbf{1}}=\mathbf{x}_{\mathbf{n}}+\mathrm{h}\left(\mathbf{A}\left(\frac{\mathbf{x}_{\mathbf{n}}+\mathbf{x}_{\mathbf{n}+\mathbf{1}}}{\mathbf{2}}\right)+\mathbf{B} \eta\left(\mathrm{t}_{\mathrm{n}}+\frac{\mathrm{h}}{2}-a\right)\right)
$$

or

$$
\mathbf{x}_{\mathbf{n}+\mathbf{1}}=\left(\mathbf{I}-\frac{\mathbf{A h}}{\mathbf{2}}\right)^{-1}\left[\left(\mathbf{I}+\frac{\mathbf{A h}}{\mathbf{2}}\right) \mathbf{x}_{\mathbf{n}}+\mathrm{h} \mathbf{B} \eta\left(\mathrm{t}_{\mathrm{n}}+\frac{\mathrm{h}}{2}-a\right)\right],
$$

where,

$$
\eta\left(\mathrm{t}_{\mathrm{n}}+\frac{\mathrm{h}}{2}-a\right)= \begin{cases}\varphi\left(t_{n}+\frac{h}{2}-a\right), & \text { if } t_{n}+\frac{h}{2}-a \leq a, \\ \frac{\left(t_{n}+\frac{h}{2}-a-t_{n-\left\lceil m a-\frac{1}{2}\right\rceil}\right) \mathbf{x}_{\mathbf{n}-\left\lfloor\mathbf{m a}-\frac{1}{2}\right\rfloor}+\left(t_{n-\left\lfloor m a-\frac{1}{2}\right\rfloor}-t_{n}+\frac{h}{2}-a\right) \mathbf{x}_{\mathbf{n}-\left\lceil\mathbf{m a}-\frac{1}{2}\right\rceil}}{h}, & \text { if } t_{n}+\frac{h}{2}-a>a\end{cases}
$$

with $h=\frac{1}{m}=0.001$.

The following tables show the numerical results of the ruin probability obtained using the three methods mentioned above. 


\begin{tabular}{|l|l|l|l|}
\hline $\mathrm{u}$ & INLAP & RK Method & Formula (15) \\
\hline \hline 0.00 & 0.9211318044 & 0.9211318044 & 0.9211318044 \\
\hline 0.50 & 0.8296021223 & 0.8296070412 & 0.8296070412 \\
\hline 1.00 & 0.6890087128 & 0.6890225081 & 0.6890225081 \\
\hline 1.50 & 0.5293799043 & 0.5298745537 & 0.5353992073 \\
\hline 2.00 & 0.3997806311 & 0.4005676986 & 0.4011025729 \\
\hline 2.50 & 0.3002514839 & 0.3014735340 & 0.3004907576 \\
\hline 3.00 & 0.2250062823 & 0.2271458282 & 0.2251155505 \\
\hline 3.50 & 0.1686190367 & 0.1721385552 & 0.1686471777 \\
\hline 4.00 & 0.1264085770 & 0.1322763643 & 0.1263432889 \\
\hline 4.50 & 0.0946434736 & 0.1131940957 & 0.0946509515 \\
\hline 5.00 & 0.0708798766 & 0.0977458827 & 0.0709083844 \\
\hline 5.50 & 0.0530743003 & 0.0850289664 & 0.0531214607 \\
\hline 6.00 & 0.0397974253 & 0.0747956981 & 0.0397962494 \\
\hline 6.50 & 0.0298436284 & 0.0670632756 & 0.0298135510 \\
\hline 7.00 & 0.0223536491 & 0.0620170348 & 0.0223348991 \\
\hline 7.50 & 0.0162816644 & 0.0600509316 & 0.0167321354 \\
\hline 8.00 & 0.0121226907 & 0.0618432334 & 0.0125346456 \\
\hline 8.50 & 0.0096039772 & 0.0748338355 & 0.0093898223 \\
\hline 9.00 & 0.0070965290 & 0.1063997278 & 0.0070334325 \\
\hline 9.50 & 0.0057058334 & 0.1677112474 & 0.0052673952 \\
\hline 10.00 & -0.0006008148 & 0.2784554477 & 0.0039430948 \\
\hline & & & \\
\hline 100 &
\end{tabular}

Table 1: Threshold 1: $\psi_{1}(u)$ with $a=1.0$ 


\begin{tabular}{|c|c|c|c|}
\hline $\mathrm{u}$ & INLAP & RK Method & Formula (15) \\
\hline 0.00 & 468887615 & 0.3468887615 & 034688 \\
\hline 0.50 & 5 & 966 & 5966 \\
\hline 1.00 & 0.2240 & 0.2240664631 & 0.2240664631 \\
\hline 1.50 & 0.169 & 0.1700244939 & 59303 \\
\hline 2.00 & 27379 & 0.1278641615 & 0.1279837661 \\
\hline 2.50 & 0.0959827900 & 0.0958782411 & 0.0958802785 \\
\hline 3.00 & 8 & 48 & 096 \\
\hline 3.50 & 52 & 0.0 & 170 \\
\hline 4.00 & 8 & 9561945 & 33515 \\
\hline 4.50 & 0301980376 & 0.0329317789 & 0.0302010202 \\
\hline 5.00 & 0.022 & 0.02690 & 0.02 \\
\hline 5.50 & 0.0169239044 & 3666 & 8890 \\
\hline 6.00 & 8 & & \\
\hline 6.50 & 0.0097050071 & 0.0129458974 & 0.0095128860 \\
\hline 7.00 & 0.0070565343 & 0.0092761645 & 0.0071266497 \\
\hline 7.50 & 0.0053980947 & 0.005 & 39924 \\
\hline 8.00 & 0.0029420257 & 0.0027 & 0.0039997697 \\
\hline 8.50 & 0.0033720732 & -0.0021464583 & 0.0029965034 \\
\hline 9.00 & 0.0015506744 & -0.0076791139 & 0.0022449363 \\
\hline 9.50 & 0.0009362698 & -0.0150272394 & 0.0016819575 \\
\hline 10.00 & 0.0032184124 & -0.0260630940 & 0.0012603061 \\
\hline
\end{tabular}

Table 2: Threshold 1: $\psi_{2}(u)$ with $a=1.0$ 


\begin{tabular}{|l|l|l|l|}
\hline $\mathrm{u}$ & INLAP & RK Method & Formula (15) \\
\hline \hline 0.00 & 0.1124110993 & 0.1124110993 & 0.1124110993 \\
\hline 0.50 & 0.0711387992 & 0.0720914189 & 0.0720914189 \\
\hline 1.00 & 0.0452700257 & 0.0462213226 & 0.0462213226 \\
\hline 1.50 & 0.0286850333 & 0.0296224339 & 0.0296224339 \\
\hline 2.00 & 0.0180510283 & 0.0189721797 & 0.0189721797 \\
\hline 2.50 & 0.0112339854 & 0.0121387152 & 0.0121387152 \\
\hline 3.00 & 0.0068656802 & 0.0077541968 & 0.0077541968 \\
\hline 3.50 & 0.0040682554 & 0.0049409821 & 0.0049409821 \\
\hline 4.00 & 0.0022786260 & 0.0031359546 & 0.0031359546 \\
\hline 4.50 & 0.0011358857 & 0.0019778046 & 0.0019778046 \\
\hline 5.00 & 0.0004084110 & 0.0012347072 & 0.0012347072 \\
\hline 5.50 & -0.0000463724 & 0.0009088316 & 0.0008124382 \\
\hline 6.00 & -0.0003241301 & 0.0006678714 & 0.0004977536 \\
\hline 6.50 & -0.0004905462 & 0.0004892724 & 0.0003049568 \\
\hline 7.00 & -0.0005879402 & 0.0003566159 & 0.0001868366 \\
\hline 7.50 & -0.0006433725 & 0.0002579009 & 0.0001144683 \\
\hline 8.00 & -0.0006729364 & 0.0001843240 & 0.0000701307 \\
\hline 8.50 & -0.0006861687 & 0.0001050259 & 0.0000429666 \\
\hline 9.00 & -0.0006893873 & 0.0000546916 & 0.0000263240 \\
\hline 9.50 & -0.0006872416 & 0.0000228227 & 0.0000161277 \\
\hline 10.00 & -0.0006800890 & 0.0000026441 & 0.0000098808 \\
\hline & 0.04 \\
\hline
\end{tabular}

Table 3: Threshold 1: $\psi_{1}(u)$ with $a=5.0$ 


\begin{tabular}{|l|l|l|l|}
\hline $\mathrm{u}$ & INLAP & RK Method & Formula $(15)$ \\
\hline \hline 0.00 & 0.0125018009 & 0.0125018009 & 0.0125018009 \\
\hline 0.50 & 0.0081177354 & 0.0080160714 & 0.0080160714 \\
\hline 1.00 & 0.0052614808 & 0.0051380803 & 0.0051380803 \\
\hline 1.50 & 0.0034384131 & 0.0032916545 & 0.0032916545 \\
\hline 2.00 & 0.0022772551 & 0.0021071074 & 0.0021071074 \\
\hline 2.50 & 0.0015403628 & 0.0013472388 & 0.0013472388 \\
\hline 3.00 & 0.0010761619 & 0.0008598552 & 0.0008598552 \\
\hline 3.50 & 0.0007862449 & 0.0005473056 & 0.0005473056 \\
\hline 4.00 & 0.0006074309 & 0.0003469348 & 0.0003469348 \\
\hline 4.50 & 0.0005011559 & 0.0002185416 & 0.0002185416 \\
\hline 5.00 & 0.0004406571 & 0.0001363320 & 0.0001363320 \\
\hline 5.50 & 0.0004101396 & 0.0001003385 & 0.0000895466 \\
\hline 6.00 & 0.0003991127 & 0.0000737371 & 0.0000548635 \\
\hline 6.50 & 0.0003991127 & 0.0000540386 & 0.0000336144 \\
\hline 7.00 & 0.0004079342 & 0.0000394291 & 0.0000205957 \\
\hline 7.50 & 0.0004200935 & 0.0000285817 & 0.0000126197 \\
\hline 8.00 & 0.0004348755 & 0.0000205222 & 0.0000077330 \\
\hline 8.50 & 0.0004515648 & 0.0000118862 & 0.0000047391 \\
\hline 9.00 & 0.0004688501 & 0.0000064647 & 0.0000029049 \\
\hline 9.50 & 0.0004863143 & 0.0000030927 & 0.0000017811 \\
\hline 10.00 & 0.0005044341 & 0.0000010186 & 0.0000010926 \\
\hline
\end{tabular}

Table 4: Threshold 1: $\psi_{2}(u)$ with $a=5.0$ 
From the above tables, we can see that $\psi_{1}(u)>\psi_{2}(u)$. Intuitively this is obvious, since $\beta_{1}=1>0.1=\beta_{2}$. When we use (15) to do the numerical calculations, the infinity summation in (16) is replaced by a finite summation. The truncation error makes that the method yields inaccurate results, in particular, when the values of $u$ are large. It is well known that the numerical inversion of Laplace transform is not stable and converges very slowly in some cases. It is also known that the RungeKutta method to solve the delay differential equation numerically is not stable. In tables 3 and 4, the computation involves many exponential functions, the cumulative errors cause the numerical results obtained by INLAP to deviate considerably from those obtained from (15). The reason why the RK method looks good in tables 3 and 4 but poor in tables 1 and 2 is that the results by RK are exact for $0 \leq u \leq 5$ in tables 3 and 4 while they are exact only for $0 \leq u \leq 1$ in tables 1 and 2 . Another reason is that we used step size 1 in tables 1 and 2 but 5 in tables 3 and 4 in our computation. For example, to calculate the result of $u=10$ from $u=1$ in tables 1 and 2 , there are 9 computation steps (the result for $u=1$ is exact). On the other hand, to do this in tables 3 and 4, the computation has to go through only 1 step from $u=5$ (the result for $u=5$ is exact), which will cause less error. Notice that unreasonable numerical results, negative probabilities, may be produced both by inverting the Laplace transform and by using the 1-stage Runge-Kutta methods of order 2. The numerical results obtained by using formula (15) are all reasonable.

To investigate the extension of the results in this paper to the case of more general claim sizes is an interesting further research problem. It is possible to obtain similar results, but the problem becomes very complex since the number of independent equations becomes large if we replace the exponential distribution by a hyper-exponential 
one. The Erlang distribution will be even more complex.

Acknowledgments: The authors would like to thank the referee for the helpful comments and suggestions. This research was supported by the Research Grants Council of the Hong Kong Special Administrative Region, China (Project No. HKU $7540 / 08 \mathrm{H})$

\section{References}

[1] Albrecher, H., Boxma, O.J. A ruin model with dependence between claim sizes and claim intervals. Insurance: Mathematics and Economics, 2004, 35, 245-254.

[2] Asmussen, S. Ruin Probabilities. World Scientific, Singapore, 2000.

[3] Bowers, N. L., Gerber, H. U., Hickman, J. C., Jones, D. A. and Nesbitt, C.J. Actuarial Mathematics. $2^{\text {nd }}$ edition, The Society of Actuaries, Schaumburg, IL, 1997.

[4] Dhaene, J. and Denuit, M. The safest dependence structure among risks, Insurance: Mathematics and Economics, 1999, 25, 11-21.

[5] Dhaene, J., Denuit, M., Goovaerts, M. J., Kaas, R. and Vyncke, D. The concept of comonotonicity in actuarial science and finance: theory, Insurance: Mathematics and Economics, 2002a, 31, 3-33. 
[6] Dhaene, J., Denuit, M., Goovaerts, M. J., Kaas, R. and Vyncke, D. The concept of comonotonicity in actuarial science and finance: applications, Insurance: Mathematics and Economics, 2002b, 31, 133-161.

[7] Dhaene, J. and Goovaverts, M. J. Dependence of risks and stop-loss order, ASTIN Bulletin, 1996, 26, 201-212.

[8] Driver, R.D. Ordinary and Delay Differential Equations. Springer-Verlag, New York, 1977.

[9] Bellen,A., Zennaro, M. Numerical Methods for Delay Differential Equations. Oxford University Press, New York, 2003.

[10] Tsoi, A.C. Explicit solution of a class of delay-differential equations. International Journal of Control, 1975, 21(1), 39-48. 